\title{
A cultura e os hábitos familiares da prática de atividades físicas e as tendências à obesidade
}

\author{
Family culture and habits of physical activity and tendencies to obesity
}

\author{
Liana Fátima Fuga" \\ Unioeste-Universidade Estadual do Oeste do Paraná- Centro de Ciências Sociais Aplicadas, Cascavel, Paraná, Brasil. \\ *Autor para correspondência. E-mail: lianaffuga@gmail.com
}

\begin{abstract}
Resumo: Esta pesquisa procura, de forma exploratória, avaliar a seguinte questão: existem relações entre aspectos culturais e hábitos familiares relacionados às atividades físicas e as tendências à obesidade na fase adulta? O objetivo é relacionar os hábitos e frequência de atividades físicas e as tendências a obtenção de índices elevados de obesidade na fase atual do indivíduo, avaliados a partir do IMC (Índice de Massa Corpórea). Especificamente, procura-se avaliar se aspectos culturais das famílias e os hábitos ao longo da vida do indivíduo permanecem ao longo do tempo. Também, se essa relação entre os hábitos e outros aspectos sócio econômicos influenciam na condição atual, a partir do indicador de referência. A pesquisa foi realizada em 2019 e 2020 na região oeste do Paraná e utiliza amostra não probabilística, a partir de dados primários. Os dados foram processados com o software SPSS e utilizou-se estatística descritiva para avaliar os resultados. A principal metodologia, no entanto, foi a utilização do cruzamento de dados (crosstabs), avaliando as relações propostas. Os resultados evidenciam relações entre a cultura e os hábitos ao longo da vida do indivíduo com sua situação de obesidade a partir da estimativa do IMC. Notadamente, a frequência da realização de atividades físicas desde a infância e a sua permanência na fase adulta, propiciam condições de melhores condições de saúde, a partir do indicador estabelecido. Relações envolvendo renda familiar não se mostraram relevante no processo relacionados ao hábitos e cultura relacionados aos exercícios. As respostas evidenciam que a frequência das atividades propicia também a percepção de bem-estar físico e mental. A partir desses resultados propõe-se que o tema esteja em evidência na educação fundamental.
\end{abstract}

Palavras-chave: cultura, hábitos familiares, atividades físicas.

\begin{abstract}
This research seeks, in an exploratory way, to evaluate the following question: are there relations between cultural aspects and family habits related to physical activities and tendencies to obesity in adulthood? The objective is to relate the habits and frequency of physical activities and the tendencies to obtain high levels of obesity in the current phase of the individual, assessed from the BMI (Body Mass Index). Specifically, it seeks to assess whether cultural aspects of families and habits over the life of the individual remain over time. Also, if this relationship between habits and other socioeconomic aspects influences the current condition, from the reference indicator. The research was carried out in 2019 and 2020 in the western region of Parana and uses a non-probabilistic sample, based on primary data. The data were processed with the SPSS software and descriptive statistics were used to evaluate the results. The main methodology, however, was the use of data crossing (crosstabs), evaluating the proposed relationships. The results show relationships between culture and habits throughout the individual's life with their obesity situation based on the BMI estimate. Notably, the frequency of physical activities since childhood and their stay in adulthood, provide conditions for better health conditions, based on the established indicator. Relationships involving family income were not relevant in the process related to habits and culture related to exercises. The answers show that the frequency of activities also provides the perception of physical and mental well-being. From these results, it is proposed that the theme be in evidence in elementary education.
\end{abstract}

Keywords: culture, family habits, physical activities.

\section{Introdução}

Uma das mais importantes epidemias que vem despontando como o mal do século XXI é a obesidade. No Brasil a prevalência da doença entre a população em todas as faixas etárias está tornando-se umas das principais preocupações no âmbito da saúde pública no País. Dados do IBGE (2015) mostram que 56,9\% da população brasileira se encontram com sobrepeso e 20,8\% estão obesas. Esses dados são preocupantes e evidenciam a necessidade cada vez maior de aprofundar o entendimento deste fenômeno, o qual tem se agravado consideravelmente nas últimas décadas. 
É notório o fato de que atualmente existem mais pessoas obesas no planeta do que famintos. A incidência da obesidade vem aumentando em todo o mundo. Segundo a Organização Mundial da Saúde (OMS, 2015), a obesidade se caracteriza como o acúmulo anormal ou excessivo de gordura que pode ser prejudicial à saúde, sendo sua causa atribuída a um desequilíbrio energético entre as calorias gastas e consumidas.

De acordo com Fisberg (1995), as evidências na contribuição dos fatores ambientais com a obesidade são muito claras. As variações na gordura corporal humana são causadas por um complexo de variáveis genéticas, nutricionais, pelo consumo de energia, psicológica e sociais. O consumo de energia está diretamente ligado ao gasto calórico, a falta de atividade física tanto na fase da infância quanto na vida adulta resulta no aumento do tecido adiposo.

Associado às alterações nas rotinas de alimentação e nos hábitos de vida da população, tidas como principais responsáveis pelo aumento da incidência de obesidade, a influência que a família pode exercer no processo de desenvolvimento da obesidade desde a infância demonstra que o IMC (índice de massa corporal) dos pais é um forte preditor de obesidade para os filhos, sugerindo que nesse processo ocorre uma interação entre fatores ambientais e genéticos.

Devido às necessidades da família contemporânea, a mulher cada vez mais contribui com a renda familiar. Por consequência, quando não estão na escola, as crianças ficam em casa sozinhas com mais acesso aos alimentos e propensas ao sedentarismo. A violência também contribui para a diminuição de atividade física, pois impede que as crianças brinquem livremente nos parques e ruas. A ausência dos pais contribui com a carência afetiva e, para compensar, muitas vezes eles cedem aos desejos das crianças aos alimentos industrializados e ao uso de eletrônicos.

De acordo com Glasenapp (2008) com a predominância do sedentarismo, associados à falta de tempo, as famílias perderam hábitos saudáveis, como passear ao ar livre e praticar atividade física para ir a shoppings, cinemas e restaurantes. Quando estes hábitos são excessivos, se tornam prejudiciais à saúde.

No contexto familiar, a obesidade se instala como uma doença crônica, que, devido à associação de mais doenças a ela, tais como hipertensão, diabetes e doenças cardiovasculares e/ou circulatórias, coloca em risco a vida do integrante familiar afetado (Ministério da Saúde, 2014). Nesse sentido, a obesidade se constitui em um fator estressor no ciclo vital de desenvolvimento da família e do indivíduo, exigindo, por parte de seus membros, a mobilização em torno de ajustes na estrutura familiar e nos papéis desempenhados por cada um.

A família é considerada um sistema aberto, em constante transformação, cujo principal papel é a proteção psicossocial de seus membros e a transmissão de sua cultura aos seus descendentes (Minuchin, 1990). Então, para que se possa compreender o fenômeno da obesidade e a sua evolução, torna-se necessária uma abordagem ampliada, em que o foco não esteja apenas no indivíduo afetado, mas em todo o seu contexto social, tendo a família um papel fundamental no processo de desenvolvimento humano e de socialização.

Este estudo foi feito com o intuito de compreender como a cultura e os hábitos familiares da prática de atividades físicas contribuem para tendências à obesidade. Considera-se que a relevância deste estudo está na possibilidade de compreensão acerca de como os padrões relacionais familiares podem influenciar no desenvolvimento desta enfermidade. O conhecimento obtido sobre esses padrões é um importante recurso de intervenção para os profissionais das áreas da saúde no contexto da doença.

Diante desse quadro em que se encontra a população, surge a questão: qual a influência dos fatores familiares e culturais nos hábitos de realização de atividades físicas e suas relações com o IMC-Î́ndice de Massa Corporal?

Para responder a esta questão de pesquisa, foram aplicados 104 questionários à população do Oeste do Paraná, entre novembro de 2019 e janeiro de 2020, a partir de amostra não probabilística, por conveniência, cujos resultados foram processados com Estatística Descritiva e Cruzamento de Dados, visando os seguintes objetivos:

O objetivo geral é relacionar os hábitos e frequência de atividades físicas e as tendências a obtenção de índices elevados de obesidade na fase atual do indivíduo, avaliados a partir do IMC (Índice de Massa Corpórea). Especificamente, procura-se avaliar se aspectos culturais das famílias e os hábitos ao longo da vida do indivíduo permanecem ao longo do tempo. Também, se essa relação entre os hábitos e outros aspectos sócio econômicos influenciam na condição atual, a partir do indicador de referência.

\section{Material e métodos}

A obesidade é provavelmente uma das enfermidades mais antigas do homem. O homem pré-histórico ingeria enormes quantidades de alimento com o objetivo de armazenar energia para sobreviver em um meio 
inóspito. Segundo Fisberg (1995), nas sociedades antigas as pessoas obesas eram associadas ao sucesso e ao poder. Na idade média o padrão estético era as formas mais arredondadas. Tudo se modifica com a chegada da busca por um corpo magro e atlético. Surgem então diversos estudos associando o peso em excesso a doenças, sendo a obesidade considerada uma enfermidade.

Até pouco tempo atrás, o grande problema da saúde pública era a desnutrição. As crianças com excesso de peso eram consideradas bem alimentadas e com muita saúde. Nos dias atuais, de acordo com a pesquisa de Glasenapp (2008), a preocupação reverteu-se para a obesidade. A desnutrição ainda é preocupante, mas está focada nas regiões menos desenvolvidas do País. Seguindo também este raciocínio, Fisberg (1995) evidenciou em seus estudos que a questão socioeconômica não interfere tanto nas questões de obesidade, mas é possível verificar que as regiões menos industrializadas o problema continua sendo a desnutrição.

O fenômeno da obesidade origina-se por diversas causas que atuam em conjunto, por isso é tão difícil encontrar um tratamento eficaz. Tais causas, segundo Glasenapp (2008) podem estar relacionadas a fatores internos e externos. Os internos são genéticos e metabólicos. Os fatores genéticos ou hereditários são os herdados dos pais pelas crianças. Quando os pais são obesos, há muita chance dos filhos se tornarem obesos também. Já os fatores metabólicos estão relacionados ao funcionamento do organismo. Algumas crianças têm mais propensão à obesidade do que outras. Já os fatores externos relacionam-se ao ambiente em que a criança está inserida. Abrangem aspectos psicológicos, hábitos alimentares, e atividade física, todos associados à influência sociocultural.

Seguindo esta linha de raciocínio, obesidade é a consequência do desequilíbrio no balanço entre a energia ingerida e aquela gasta para a manutenção dos processos vitais e trabalho desenvolvido. A obesidade, portanto, pode ser desencadeada quando há excesso na ingestão e ausência de exercícios físicos que promovam o gasto energético, ou quando ocorre variações negativas no metabolismo. Segundo Fisberg (1995), a alta prevalência da obesidade infantil e suas consequências no desenvolvimento psicossocial contribuem para que os riscos inerentes persistam na fase adulta.

A obesidade afeta tanto a saúde física quanto a emocional. Os pais, além de buscar uma solução para a obesidade com uma alimentação mais saudável e a prática regular de exercícios físicos, podem colaborar e se beneficiar do atendimento psicológico, analisando e compreendendo as razões emocionais envolvidas no surgimento e na evolução da obesidade do seu filho.

Considera-se relevante destacar também que a família contemporânea se encontra inserida em um contexto sociocultural cujos padrões estéticos envolvem a valorização da imagem corporal e a busca por corpos cada vez mais magros (Goldenberg\& Ramos, 2007; Vasques, Martins \& Azevedo, 2004). Assim, a insatisfação com o próprio corpo devido ao excesso de peso é capaz de gerar sofrimento para os sujeitos obesos pelo fato de não se enquadrarem nestes padrões, e deve ser considerada como um fator ambiental significativo nesse cenário. Em resumo, entende-se que as características do ambiente familiar podem ser tanto um importante fator potencialmente de risco para o desenvolvimento da doença, como um fator potencialmente de proteção, o que torna necessário que esse aspecto seja observado nos processos de intervenção em famílias com membros obesos.

As investigações apontam o ambiente familiar e a qualidade das relações na família como um importante fator influenciador no ganho excessivo de peso. A pesquisa realizada por Crossman, Sullivan, e Benin (2006) nos Estados Unidos teve por objetivo investigar se o ambiente familiar dos adolescentes tem efeitos em longo prazo sobre o seu peso. Para o estudo de abordagem quantitativa foi utilizado um recorte dos dados da pesquisa National Longitudinal StudyofAdolescent Health, que foi correlacionado com aspectos da interação familiar, como a percepção do controle dos pais, a percepção de cuidado parental e a estrutura familiar. Um dos resultados da pesquisa apontou que o ambiente familiar dos adolescentes exerce influência em seu peso até a idade adulta.

Os estudos enquadrados na hereditariedade foram desenvolvidos com foco na difusão da obesidade entre as diferentes gerações da família e na prevalência da doença dentro do sistema familiar. A pesquisa desenvolvida por Moira, Power e Li (2010) na Grã-Bretanha tinha por objetivo determinar se a prevalência da obesidade infantil mudou entre a geração dos pais nascidos em 1958 e seus descendentes e se essas mudanças teriam contribuído para a tendência de obesidade infantil. Os pesquisadores utilizaram recortes dos dados da pesquisa longitudinal British BirthCohort para estabelecer relações entre os IMCs (índices de massa corporal) apresentados por pais e seus respectivos filhos. Os resultados do estudo evidenciaram que a prevalência de sobrepeso/obesidade aumentou em mais de $50 \%$ entre as gerações. Os principais fatores preditivos do aumento no peso das crianças foram, além do IMC dos pais, o trabalho materno em tempo integral. Tal constatação poderia ser explicada pelas funções e papéis tradicionalmente atribuídos pela sociedade às mulheres dentro do contexto familiar. 
Na pesquisa de Fox et al. (2013) também foram encontrados fatores que associam a presença da obesidade entre as gerações. $\mathrm{O}$ objetivo do estudo era explorar a associação entre o histórico de obesidade dos pais e o desenvolvimento da doença em seus descendentes ao longo do tempo. Os autores utilizaram dados de três gerações para comparar o desenvolvimento da obesidade ao longo de sessenta anos. Os achados mostraram que a história familiar de obesidade é um fator de risco importante para o aumento do IMC entre os filhos de pais afetados, sendo que essa associação ocorre com maior força na terceira geração. A pesquisa mostrou que indivíduos com um dos pais obesos não apresentaram maior risco de desenvolver obesidade, enquanto aqueles indivíduos em que os dois pais eram obesos apresentaram um aumento de 37\% no risco de desenvolvimento de obesidade. Esses dados se relacionam com os da pesquisa desenvolvida por Mascarenhas et al. (2013) em contexto brasileiro, que encontrou dados semelhantes, em que foi possível verificar que o risco de desenvolvimento de sobrepeso/obesidade nos filhos é 2,65 vezes maior quando ambos os pais apresentam sobrepeso.

Estas pesquisas podem evidenciar uma explicação para a epidemia de obesidade no mundo, uma vez que as gerações mais recentes têm vivido com maior intensidade os efeitos desta associação. Nessa visão, cabe também o questionamento a respeito das consequências e efeitos em longo prazo da transmissão da obesidade, já que o aumento na prevalência da doença no contexto familiar pode acarretar uma naturalização do processo de ganho excessivo de peso dentro da família, de maneira a dificultar a percepção acerca dos riscos envolvidos e da necessidade de tratamento para os indivíduos afetados. Muitas vezes a obesidade não é considerada uma doença para as famílias que convivem com esta condição há muito tempo.

Tanto a pesquisa de Kelly et al. (2014), realizada em contexto internacional, como a de Geremia et al. (2015), realizada em contexto brasileiro, encontraram resultados semelhantes e identificaram fortes associações entre a presença de excesso de peso nas mães e o excesso de peso nas crianças, sugerindo que a tendência ao desenvolvimento de obesidade está predominantemente associada à linha materna. Sobre a influência materna no peso das crianças, Mascarenhas et al. (2013) também identificaram que a obesidade materna é um fator de risco para o desenvolvimento de sobrepeso/obesidade nos filhos, ocorrendo tanto nos de sexo masculino quanto nos de sexo feminino.

No conjunto das investigações observadas, o papel da mãe é considerado como um dos principais protagonistas pela transmissão da obesidade entre as gerações. Considera-se importante refletir sobre o papel materno na sociedade, bem como no contexto da produção científica, o qual, necessariamente, precisa ser analisado na inter-relação com os demais aspectos que convergem no surgimento da obesidade, evitando, assim, a excessiva responsabilização da figura materna pelo problema. Segundo Fisberg (1995), empiricamente observa-se uma predisposição genética à obesidade. Provavelmente a influência genética na obesidade humana está relacionada às características no metabolismo do indivíduo.

Já um estudo realizado na Suécia abordou o problema sob a ótica das questões socioeconômicas envolvidas na transmissão da obesidade. O objetivo do estudo era investigar o impacto da trajetória educacional dos avós e dos pais no status de sobrepeso e obesidade nos filhos adultos. Para isso, os autores correlacionaram os dados socioeconômicos de duas pesquisas longitudinais com o IMC de três gerações dessas famílias. Os resultados obtidos sugerem que tanto a educação dos avós como a dos pais têm impacto sobre o excesso de peso/obesidade de seus filhos adultos. Tanto para o sexo feminino como para o sexo masculino, as famílias cujos pais sempre pertenceram a um grupo socioeconômico desfavorecido obtiveram cerca de duas vezes mais chances de ter excesso de peso ou obesidade, em comparação com as famílias que sempre pertenceram ao grupo socioeconômico favorecido (Baratieri, 2005).

A pesquisa que foi realizada na Suécia, que é considerado um país desenvolvido, mas resultados semelhantes também podem ser encontrados em países menos desenvolvidos como o Brasil, a Argentina e o Chile. Nesses países, pesquisas indicaram que a presença das maiores taxas de excesso de peso e obesidade é encontrada entre a população de baixa renda e baixa escolaridade (Ministério da Saúde, 2014). Esses achados parecem trazer à tona um paradoxo, uma vez que se percebe entre a população de baixa renda a presença cada vez maior da obesidade quando comparada à desnutrição, o que aponta não para a carência de alimentos, mas, sim, para uma má qualidade nutricional destes (Baratieri, 2005). Nesse contexto paradoxal, a obesidade se apresenta como um grave problema de saúde pública em nível mundial, desafiando a produção científica no desenvolvimento de mais pesquisas que auxiliem na compreensão acerca das influências sobre os padrões alimentares dessas populações na perspectiva da hereditariedade.

Segundo Glasenapp (2008) em sua pesquisa na cidade de Joinville, SC, investigou os hábitos alimentares e a prática esportiva de um grupo de educandos da educação infantil e séries iniciais do ensino fundamental, como consequência para a elevação dos índices de obesidade infantil. Os dados coletados apontaram para os maus hábitos alimentares e para a ausência de prática desportiva entre as crianças, que, associados, vêm elevando a incidência da obesidade. 
Há evidências na literatura que a ingestão alimentar não é regulada da mesma forma nas diferentes intensidades da atividade física. Quanto mais intensa é a atividade física, maior o gasto energético e como consequência a perda de peso. Segundo Fisberg (1995), as atividades motoras, como um dos fatores de intervenção, podem proporcionar mudanças significativas quanto a obesidade, promovendo a redução no número de células adiposas. Sendo assim, torna-se importante intervir o quanto antes e incentivar nas crianças a prática de exercícios físicos, pois é nesta fase que estão sendo desenvolvidos potenciais metabólicos influenciadores capazes de estabelecer padrões de obesidade que se manterão durante a vida adulta.

A partir desses dados, podemos verificar a necessidade desta discussão no âmbito escolar, pois a difícil solução do problema da obesidade sugere que para a prevenção e um tratamento eficaz deve haver um trabalho conjunto entre famílias e escolas, uma reeducação alimentar e incentivo a atividade física, para que assim os hábitos saudáveis retornem nas famílias.

É primordial que o trabalho de prevenção seja feito nas escolas e que as famílias mudem os hábitos que são prejudiciais à sua saúde. Os pais possuem papel importante no sentido de colaborar com a escola e orientar seus filhos a uma alimentação mais saudável e a prática regular de exercícios físicos.

\section{Resultados e discussão}

Aplicada a pesquisa entre novembro de 2019 e fevereiro de 2020, os resultados evidenciam que, de fato, em média a população está com pesos acima do recomendado. Somando-se os resultados correspondentes a "Levemente acima do peso" com "Obesidade Grau I", conforme mostra o Quadro 1, temos que entre as mulheres, o peso aumenta com a idade, pois $81,8 \%$ das que têm idade superior a 51 anos encontram-se nesta condição. Por outro lado, as que tem idade entre 36 e 50 anos, somente $48 \%$ estão nesses grupos. Reafirmando essa condição, as mulheres com idade até 35 anos, somente $28,6 \%$ encontram-se nesta condição. Os resultados mostram a necessidade de as mulheres praticarem atividades físicas, na medida em que avançam para faixa etárias mais altas.

O esperado é que os mesmos resultados ocorressem para o sexo masculino. Observa-se que, entre os homens, 66,7\% com mais de 51 anos encontram-se nessa condição (Levemente acima do peso e Obesidade Grau I). Esse percentual é inferior ao encontrado no sexo feminino, que atingiu 81,8\%. Todavia, na faixa entre 36 e 50 anos, são $66,6 \%$ entre os homens e $48,8 \%$ entre as mulheres. Com as idades entre 21 e 35 anos, são $45,5 \%$ entre os homens e somente $28,6 \%$ entre as mulheres. Observa-se que o padrão entre os homens mais novos e as mulheres mais novas não e repete, sendo que os homens estão obesos mais cedo. Praticamente a metade deles $(45,5 \%)$ está levemente acima do peso ou obesidade de Grau I.

\% em Perfil Socioeconômico-Faixa Etária

\begin{tabular}{|c|c|c|c|c|c|c|c|}
\hline \multirow{2}{*}{\multicolumn{3}{|c|}{ Perfil Socioeconômico-sexo }} & \multicolumn{4}{|c|}{ Classificação IMC } & \multirow[b]{2}{*}{ Total } \\
\hline & & & $\begin{array}{c}\text { Abaixo do } \\
\text { peso }\end{array}$ & $\begin{array}{c}\text { Levemente } \\
\text { acima do } \\
\text { peso }\end{array}$ & $\begin{array}{c}\text { Obesidade } \\
\text { grau I }\end{array}$ & Peso Ideal & \\
\hline \multirow[t]{6}{*}{ Feminino } & Perfil Socioeconômico- & Até 18 anos & & & & $100,0 \%$ & $100,0 \%$ \\
\hline & & Entre 21 e 35 anos & $19,0 \%$ & $28,6 \%$ & & $52,4 \%$ & $100,0 \%$ \\
\hline & & Entre 36 e 50 anos & $4,0 \%$ & $40,0 \%$ & $8,0 \%$ & $48,0 \%$ & $100,0 \%$ \\
\hline & & Entre 51 e 65 anos & & $27,3 \%$ & $54,5 \%$ & $18,2 \%$ & $100,0 \%$ \\
\hline & & Mais de 65 anos & & $20,0 \%$ & $40,0 \%$ & $40,0 \%$ & $100,0 \%$ \\
\hline & Total & & $7,9 \%$ & $31,7 \%$ & $15,9 \%$ & $44,4 \%$ & $100,0 \%$ \\
\hline \multirow[t]{6}{*}{ Masculino } & Perfil Socioeconômico- & Até 18 anos & & & & $100,0 \%$ & $100,0 \%$ \\
\hline & Faixa Etária & Entre 21 e 35 anos & & $27,3 \%$ & $18,2 \%$ & $54,5 \%$ & $100,0 \%$ \\
\hline & & Entre 36 e 50 anos & & $53,3 \%$ & $13,3 \%$ & $33,3 \%$ & $100,0 \%$ \\
\hline & & Entre 51 e 65 anos & & $16,7 \%$ & $50,0 \%$ & $33,3 \%$ & $100,0 \%$ \\
\hline & & Mais de 65 anos & & $50,0 \%$ & & $50,0 \%$ & $100,0 \%$ \\
\hline & Total & & & $34,1 \%$ & $24,4 \%$ & $41,5 \%$ & $100,0 \%$ \\
\hline \multirow[t]{6}{*}{ Total } & Perfil Socioeconômico- & Até 18 anos & & & & $100,0 \%$ & $100,0 \%$ \\
\hline & Faixa Etária & Entre 21 e 35 anos & $12,5 \%$ & $28,1 \%$ & $6,3 \%$ & $53,1 \%$ & $100,0 \%$ \\
\hline & & Entre 36 e 50 anos & $2,5 \%$ & $45,0 \%$ & $10,0 \%$ & $42,5 \%$ & $100,0 \%$ \\
\hline & & Entre 51 e 65 anos & & $21,7 \%$ & $52,2 \%$ & $26,1 \%$ & $100,0 \%$ \\
\hline & & Mais de 65 anos & & $28,6 \%$ & $28,6 \%$ & $42,9 \%$ & $100,0 \%$ \\
\hline & Total & & $4,8 \%$ & $32,7 \%$ & $19,2 \%$ & $43,3 \%$ & $100,0 \%$ \\
\hline
\end{tabular}

Quadro 1. Relação Classificação do IMC, por Faixa Etária, segmentado por Gênero. Fonte: Resultados da pesquisa. 
Observando os resultados dos que estão com peso ideal (Quadro 2), comparados os mesmos segmentados por grau de escolaridade, entre as mulheres que têm Pós-graduação, 57,6\% estão com peso ideal e entre os homens somente $41,2 \%$. Porém, ao se comprar o resultado global entre os níveis de escolaridade não se observam grandes modificações no perfil do que estão com peso ideal.

Perfil Socioeconômico-Grau de escolaridade * Classificação IMC * Perfil Socioeconômico-sexo Tabulação cruzada

$\%$ em Perfil Socioeconômico-Grau de escolaridade

\begin{tabular}{|c|c|c|c|c|c|c|c|}
\hline \multirow{2}{*}{\multicolumn{2}{|c|}{ Perfil Socioeconômico-sexo }} & & \multicolumn{4}{|c|}{ Classificação IMC } & \multirow[b]{2}{*}{ Total } \\
\hline & & & $\begin{array}{c}\text { Abaixo do } \\
\text { peso }\end{array}$ & \multirow{2}{*}{$\begin{array}{r}\begin{array}{c}\text { Levemente } \\
\text { acima do } \\
\text { peso }\end{array} \\
37,5 \%\end{array}$} & \multirow{2}{*}{$\begin{array}{l}\begin{array}{l}\text { Obesidade } \\
\text { grau I }\end{array} \\
12,5 \%\end{array}$} & \multirow{2}{*}{$\begin{array}{r}\text { Peso Ideal } \\
50,0 \%\end{array}$} & \\
\hline Feminino & $\begin{array}{l}\text { Perfil Socioeconômico- } \\
\text { Grau de escolaridade }\end{array}$ & $\begin{array}{l}\text { Ensino médio completo } \\
\text { ou incompleto }\end{array}$ & \multirow[b]{2}{*}{$13,6 \%$} & & & & $100,0 \%$ \\
\hline & & $\begin{array}{l}\text { Ensino superior } \\
\text { completo ou incompleto }\end{array}$ & & $40,9 \%$ & $22,7 \%$ & $22,7 \%$ & $100,0 \%$ \\
\hline & & $\begin{array}{l}\text { Pós-graduação } \\
\text { (especialização, } \\
\text { mestrado ou doutorado) }\end{array}$ & $6,1 \%$ & $24,2 \%$ & $12,1 \%$ & $57,6 \%$ & $100,0 \%$ \\
\hline & Total & & $7,9 \%$ & $31,7 \%$ & $15,9 \%$ & $44,4 \%$ & $100,0 \%$ \\
\hline \multirow[t]{5}{*}{ Masculino } & $\begin{array}{l}\text { Perfil Socioeconômico- } \\
\text { Grau de escolaridade }\end{array}$ & $\begin{array}{l}\text { Ensino fundamental } \\
\text { completo ou incompleto }\end{array}$ & & $100,0 \%$ & & & $100,0 \%$ \\
\hline & & $\begin{array}{l}\text { Ensino médio completo } \\
\text { ou incompleto }\end{array}$ & & $25,0 \%$ & $50,0 \%$ & $25,0 \%$ & $100,0 \%$ \\
\hline & & $\begin{array}{l}\text { Ensino superior } \\
\text { completo ou incompleto }\end{array}$ & & $31,6 \%$ & $21,1 \%$ & $47,4 \%$ & $100,0 \%$ \\
\hline & & $\begin{array}{l}\text { Pós-graduação } \\
\text { (especialização, } \\
\text { mestrado ou doutorado) }\end{array}$ & & $35,3 \%$ & $23,5 \%$ & $41,2 \%$ & $100,0 \%$ \\
\hline & Total & & & $34,1 \%$ & $24,4 \%$ & $41,5 \%$ & $100,0 \%$ \\
\hline \multirow[t]{5}{*}{ Total } & $\begin{array}{l}\text { Perfil Socioeconômico- } \\
\text { Grau de escolaridade }\end{array}$ & $\begin{array}{l}\text { Ensino fundamental } \\
\text { completo ou incompleto }\end{array}$ & & $100,0 \%$ & & & $100,0 \%$ \\
\hline & & $\begin{array}{l}\text { Ensino médio completo } \\
\text { ou incompleto }\end{array}$ & & $33,3 \%$ & $25,0 \%$ & $41,7 \%$ & $100,0 \%$ \\
\hline & & $\begin{array}{l}\text { Ensino superior } \\
\text { completo ou incompleto }\end{array}$ & $7,3 \%$ & $36,6 \%$ & $22,0 \%$ & $34,1 \%$ & $100,0 \%$ \\
\hline & & $\begin{array}{l}\text { Pós-graduação } \\
\text { (especialização, } \\
\text { mestrado ou doutorado) }\end{array}$ & $4,0 \%$ & $28,0 \%$ & $16,0 \%$ & $52,0 \%$ & $100,0 \%$ \\
\hline & Total & & $4,8 \%$ & $32,7 \%$ & $19,2 \%$ & $43,3 \%$ & $100,0 \%$ \\
\hline
\end{tabular}

Quadro 2. Relação Classificação do IMC, por Escolaridade, segmentado por Gênero. Fonte: Resultados da pesquisa.

Ao se comparar a situação de obesidade com a renda familiar mensal, conforme mostra o Quadro 3, temos que, os maiores problemas de obesidade ocorrem com famílias com renda mais baixas. Entre as mulheres, com renda familiar de até R\$ 3 mil mensais, $70 \%$ estão na condição de Obesidade Grau I e Levemente acima do peso, ou seja, em condição inadequada. Entre os homens com esse mesmo nível de renda, $40 \%$ estão nessa condição. Na medida em que a renda sobe, entre R $\$ 3$ e R $\$ 5$ mil, 50\% entre mulheres e também homens estão nessa condição. Já para níveis de renda mais alta, entre $\mathrm{R} \$ 5$ e 10 mil, 51,8\% no total entre homens e mulheres estão nessa condição. Ou seja, com rendas altas e muito altas, há uma equalização desses resultados.

Ao se avaliar sob o aspecto cultural, como as famílias compreendem a importância da realização das atividades físicas, conforme observado no Quadro 4, temos que: no total dos dois segmentos, masculino e feminino, $43,3 \%$ estão com peso ideal. São $4,8 \%$ abaixo do peso e $51,9 \%$ estão com grau de obesidade I ou levemente acima do peso. Todavia, com relação à concordância ou não com a afirmativa de prática de atividades físicas na infância e o grau de obesidade, as relações são fracas, não sendo possível dizer que há evidências dessa relação.

Conforme mostra o quadro 5 , que evidencia a classificação, quando confrontadas com a afirmativa que "atualmente continuam praticando atividades físicas regularmente", as que concordam totalmente, 53,6\% estão com peso ideal. Entre os homens, 55,6\% estão com peso ideal. Ou seja, evidencia-se a relação direta entre realização de atividades físicas e peso ideal. Já entre os que discordam da afirmativa e os que discordam totalmente, $34,6 \%$ e 33,3\% estão com peso ideal, respectivamente. Ou seja, em torno de $66 \%$ não estão com peso ideal e não praticam atividades físicas regularmente. A prática cultural de realização de atividades físicas coloca em melhores condições na classificação de peso ideal essas pessoas, tanto do sexo masculino quanto do sexo feminino. 
O quadro 6, evidencia os resultados comparando a classificação do IMC do respondente com a realização de atividades físicas pelos pais. Observa-se que em torno de $60 \%$ dos que tiveram pais que praticavam atividades físicas durante sua infância, estão com peso ideal. De alguma forma, os aspectos culturais familiares ligados à alimentação e práticas diversas, como entre elas, a prática de atividades físicas, passa de geração em geração e influencia também nas condições de saúde do indivíduo. Perfil Socioeconômico-Faixa de renda mensal familiar (Total da renda mensal dos que moram na mesma casa). * Classificação IMC * Perfil
Socioeconômico-sexo Tabulação cruzada

\% em Perfil Socioeconômico-Faixa de renda mensal familiar (Total da renda mensal dos que moram na mesma casa).

\begin{tabular}{|c|c|c|c|c|c|c|c|}
\hline \multirow{2}{*}{\multicolumn{3}{|c|}{ Perfil Socioeconômico-sexo }} & \multicolumn{4}{|c|}{ Classificação IMC } & \multirow[b]{2}{*}{ Total } \\
\hline & & & $\begin{array}{c}\text { Abaixo do } \\
\text { peso }\end{array}$ & \multirow{2}{*}{$\begin{array}{r}\begin{array}{c}\text { Levemente } \\
\text { acima do } \\
\text { peso }\end{array} \\
22,2 \%\end{array}$} & \multirow{2}{*}{$\begin{array}{r}\begin{array}{c}\text { Obesidade } \\
\text { grau I }\end{array} \\
11,1 \%\end{array}$} & \multirow{2}{*}{$\frac{\text { Peso Ideal }}{44,4 \%}$} & \\
\hline Feminino & $\begin{array}{l}\text { Perfil Socioeconômico- } \\
\text { Faixa de renda mensal }\end{array}$ & $\begin{array}{l}\text { Acima de } R \$ 20.000,00 \\
\text { mensais. }\end{array}$ & $22,2 \%$ & & & & $100,0 \%$ \\
\hline & familiar (Total da renda & $\begin{array}{l}\text { Até } R \$ 3.000,00 \\
\text { mensais. }\end{array}$ & $10,0 \%$ & $50,0 \%$ & $20,0 \%$ & $20,0 \%$ & $100,0 \%$ \\
\hline & & $\begin{array}{l}\text { De } R \$ 10.001,00 \text { a } R \$ \\
20.000,00 \text { mensais. }\end{array}$ & \multirow{3}{*}{$12,5 \%$} & $40,0 \%$ & $6,7 \%$ & $53,3 \%$ & $100,0 \%$ \\
\hline & & $\begin{array}{l}\text { De } R \$ 3.001,00 \text { a } R \$ \\
5.000,00 \text { mensais. }\end{array}$ & & $25,0 \%$ & $25,0 \%$ & $37,5 \%$ & $100,0 \%$ \\
\hline & & $\begin{array}{l}\text { De } R \$ 5.001,00 \text { a } R \$ \\
10.000,00 \text { mensais. }\end{array}$ & & $23,1 \%$ & $15,4 \%$ & $61,5 \%$ & $100,0 \%$ \\
\hline & Total & & $7,9 \%$ & $31,7 \%$ & $15,9 \%$ & $44,4 \%$ & $100,0 \%$ \\
\hline \multirow[t]{6}{*}{ Masculino } & \multirow{5}{*}{$\begin{array}{l}\text { Perfil Socioeconômico- } \\
\text { Faixa de renda mensal } \\
\text { familiar (Total da renda } \\
\text { mensal dos que moram } \\
\text { na mesma casa). }\end{array}$} & $\begin{array}{l}\text { Acima de } R \$ 20.000,00 \\
\text { mensais. }\end{array}$ & & $22,2 \%$ & $\begin{array}{r}100,0 \% \\
33,3 \%\end{array}$ & $44,4 \%$ & $\begin{array}{l}100,0 \% \\
100,0 \%\end{array}$ \\
\hline & & $\begin{array}{l}\text { Até } R \$ 3.000,00 \\
\text { mensais. }\end{array}$ & & $20,0 \%$ & $20,0 \%$ & $60,0 \%$ & $100,0 \%$ \\
\hline & & $\begin{array}{l}\text { De } R \$ 10.001,00 \text { a } R \$ \\
20.000,00 \text { mensais. }\end{array}$ & & $75,0 \%$ & & $25,0 \%$ & $100,0 \%$ \\
\hline & & $\begin{array}{l}\text { De } R \$ 3.001,00 \text { a } R \$ \\
5.000,00 \text { mensais. }\end{array}$ & & $37,5 \%$ & $12,5 \%$ & $50,0 \%$ & $100,0 \%$ \\
\hline & & $\begin{array}{l}\text { De } R \$ 5.001,00 \text { a } R \$ \\
10.000,00 \text { mensais. }\end{array}$ & & $35,7 \%$ & $28,6 \%$ & $35,7 \%$ & $100,0 \%$ \\
\hline & Total & & & $34,1 \%$ & $24,4 \%$ & $41,5 \%$ & $100,0 \%$ \\
\hline \multirow[t]{6}{*}{ Total } & \multirow{5}{*}{$\begin{array}{l}\text { Perfil Socioeconômico- } \\
\text { Faixa de renda mensal } \\
\text { familiar (Total da renda } \\
\text { mensal dos que moram } \\
\text { na mesma casa). }\end{array}$} & $\begin{array}{l}\text { Acima de } R \$ 20.000,00 \\
\text { mensais. }\end{array}$ & $11,1 \%$ & $22,2 \%$ & $\begin{array}{r}100,0 \% \\
22,2 \%\end{array}$ & $44,4 \%$ & $\begin{array}{l}100,0 \% \\
100,0 \%\end{array}$ \\
\hline & & $\begin{array}{l}\text { Até } R \$ 3.000,00 \\
\text { mensais. }\end{array}$ & $6,7 \%$ & $40,0 \%$ & $20,0 \%$ & $33,3 \%$ & $100,0 \%$ \\
\hline & & $\begin{array}{l}\text { De } R \$ 10.001,00 \text { a } R \$ \\
20.000,00 \text { mensais. }\end{array}$ & \multirow{3}{*}{$8,3 \%$} & $47,4 \%$ & $5,3 \%$ & $47,4 \%$ & $100,0 \%$ \\
\hline & & $\begin{array}{l}\text { De } R \$ 3.001,00 \text { a } R \$ \\
5.000,00 \text { mensais. }\end{array}$ & & $29,2 \%$ & $20,8 \%$ & $41,7 \%$ & $100,0 \%$ \\
\hline & & $\begin{array}{l}\text { De } R \$ 5.001,00 \text { a } R \$ \\
10.000,00 \text { mensais. }\end{array}$ & & $29,6 \%$ & $22,2 \%$ & $48,1 \%$ & $100,0 \%$ \\
\hline & \multicolumn{2}{|l|}{ Total } & $4,8 \%$ & $32,7 \%$ & $19,2 \%$ & $43,3 \%$ & $100,0 \%$ \\
\hline
\end{tabular}

Quadro 3. Relação Classificação do IMC, por Renda, segmentado por Gênero. Fonte: Resultados da pesquisa.

Desde cedo, quando ainda criança, sempre praticava atividades físicas. * Classificação IMC * Perfil Socioeconômico-sexo Tabulação cruzada

$\%$ em Desde cedo, quando ainda criança, sempre praticava atividades físicas.

\begin{tabular}{|c|c|c|c|c|c|c|c|}
\hline \multirow{2}{*}{\multicolumn{3}{|c|}{ Perfil Socioeconômico-sexo }} & \multicolumn{4}{|c|}{ Classificação IMC } & \multirow[b]{2}{*}{ Total } \\
\hline & & & $\begin{array}{c}\text { Abaixo do } \\
\text { peso }\end{array}$ & $\begin{array}{l}\text { Levemente } \\
\text { acima do } \\
\text { peso }\end{array}$ & $\begin{array}{c}\text { Obesidade } \\
\text { grau I }\end{array}$ & Peso Ideal & \\
\hline \multirow[t]{5}{*}{ Feminino } & \multirow{4}{*}{$\begin{array}{l}\text { Desde cedo, quando } \\
\text { ainda criança, sempre } \\
\text { praticava atividades } \\
\text { físicas. }\end{array}$} & Concordo totalmente. & $14,3 \%$ & $14,3 \%$ & $14,3 \%$ & $57,1 \%$ & $100,0 \%$ \\
\hline & & Concordo. & & $40,7 \%$ & $22,2 \%$ & $37,0 \%$ & $100,0 \%$ \\
\hline & & Discordo totalmente. & & $100,0 \%$ & & & $100,0 \%$ \\
\hline & & Discordo. & $18,2 \%$ & $18,2 \%$ & $9,1 \%$ & $54,5 \%$ & $100,0 \%$ \\
\hline & Total & & $7,9 \%$ & $31,7 \%$ & $15,9 \%$ & $44,4 \%$ & $100,0 \%$ \\
\hline \multirow[t]{4}{*}{ Masculino } & \multirow{3}{*}{$\begin{array}{l}\text { Desde cedo, quando } \\
\text { ainda criança, sempre } \\
\text { praticava atividades } \\
\text { físicas. }\end{array}$} & Concordo totalmente. & & $33,3 \%$ & $25,0 \%$ & $41,7 \%$ & $100,0 \%$ \\
\hline & & Concordo. & & $35,0 \%$ & $25,0 \%$ & $40,0 \%$ & $100,0 \%$ \\
\hline & & Discordo. & & $33,3 \%$ & $22,2 \%$ & $44,4 \%$ & $100,0 \%$ \\
\hline & Total & & & $34,1 \%$ & $24,4 \%$ & $41,5 \%$ & $100,0 \%$ \\
\hline \multirow[t]{5}{*}{ Total } & \multirow{4}{*}{$\begin{array}{l}\text { Desde cedo, quando } \\
\text { ainda criança, sempre } \\
\text { praticava atividades } \\
\text { físicas. }\end{array}$} & Concordo totalmente. & $9,1 \%$ & $21,2 \%$ & $18,2 \%$ & $51,5 \%$ & $100,0 \%$ \\
\hline & & Concordo. & & $38,3 \%$ & $23,4 \%$ & $38,3 \%$ & $100,0 \%$ \\
\hline & & Discordo totalmente. & & $100,0 \%$ & & & $100,0 \%$ \\
\hline & & Discordo. & $10,0 \%$ & $25,0 \%$ & $15,0 \%$ & $50,0 \%$ & $100,0 \%$ \\
\hline & Total & & $4,8 \%$ & $32,7 \%$ & $19,2 \%$ & $43,3 \%$ & $100,0 \%$ \\
\hline
\end{tabular}

Quadro 4. Relação Classificação do IMC, práticas de atividades físicas quando criança, segmentado por Gênero. Fonte: Resultados da pesquisa. 
Atualmente continuo praticando atividades físicas com certa regularidade. * Classificação IMC * Perfil Socioeconômico-sexo Tabulação cruzada

$\%$ em Atualmente continuo praticando atividades físicas com certa regularidade.

\begin{tabular}{|c|c|c|c|c|c|c|c|}
\hline \multirow{2}{*}{\multicolumn{2}{|c|}{ Perfil Socioeconômico-sexo }} & & \multicolumn{4}{|c|}{ Classificação IMC } & \multirow[b]{2}{*}{ Total } \\
\hline & & & $\begin{array}{l}\text { Abaixo do } \\
\text { peso }\end{array}$ & $\begin{array}{c}\text { Levemente } \\
\text { acima do } \\
\text { peso }\end{array}$ & $\begin{array}{c}\text { Obesidade } \\
\text { grau I }\end{array}$ & Peso Ideal & \\
\hline \multirow[t]{5}{*}{ Feminino } & \multirow{4}{*}{$\begin{array}{l}\text { Atualmente continuo } \\
\text { praticando atividades } \\
\text { físicas com certa } \\
\text { regularidade. }\end{array}$} & Concordo totalmente. & $10,7 \%$ & $35,7 \%$ & & $53,6 \%$ & $100,0 \%$ \\
\hline & & Concordo. & $4,8 \%$ & $23,8 \%$ & $38,1 \%$ & $33,3 \%$ & $100,0 \%$ \\
\hline & & Discordo totalmente. & & $25,0 \%$ & $25,0 \%$ & $50,0 \%$ & $100,0 \%$ \\
\hline & & Discordo. & $10,0 \%$ & $40,0 \%$ & $10,0 \%$ & $40,0 \%$ & $100,0 \%$ \\
\hline & Total & & $7,9 \%$ & $31,7 \%$ & $15,9 \%$ & $44,4 \%$ & $100,0 \%$ \\
\hline \multirow[t]{6}{*}{ Masculino } & Atualmente continuo & Concordo totalmente. & & $37,5 \%$ & & $62,5 \%$ & $100,0 \%$ \\
\hline & $\begin{array}{l}\text { praticando atividades } \\
\text { físicas com certa }\end{array}$ & Concordo. & & $23,1 \%$ & $23,1 \%$ & $53,8 \%$ & $100,0 \%$ \\
\hline & regularidade. & Discordo totalmente. & & $50,0 \%$ & $50,0 \%$ & & $100,0 \%$ \\
\hline & & Discordo. & & $31,3 \%$ & $37,5 \%$ & $31,3 \%$ & $100,0 \%$ \\
\hline & & $\begin{array}{l}\text { Não tenho opinião } \\
\text { formada. }\end{array}$ & & $100,0 \%$ & & & $100,0 \%$ \\
\hline & Total & & & $34,1 \%$ & $24,4 \%$ & $41,5 \%$ & $100,0 \%$ \\
\hline \multirow[t]{6}{*}{ Total } & Atualmente continuo & Concordo totalmente. & $8,3 \%$ & $36,1 \%$ & & $55,6 \%$ & $100,0 \%$ \\
\hline & $\begin{array}{l}\text { praticando atividades } \\
\text { físicas com certa }\end{array}$ & Concordo. & $2,9 \%$ & $23,5 \%$ & $32,4 \%$ & $41,2 \%$ & $100,0 \%$ \\
\hline & regularidade. & Discordo totalmente. & & $33,3 \%$ & $33,3 \%$ & $33,3 \%$ & $100,0 \%$ \\
\hline & & Discordo. & $3,8 \%$ & $34,6 \%$ & $26,9 \%$ & $34,6 \%$ & $100,0 \%$ \\
\hline & & $\begin{array}{l}\text { Não tenho opinião } \\
\text { formada. }\end{array}$ & & & & & \\
\hline & Total & & $4,8 \%$ & $32,7 \%$ & $19,2 \%$ & $43,3 \%$ & $100,0 \%$ \\
\hline
\end{tabular}

Quadro 5. Relação Classificação do IMC, práticas atuais de atividades físicas, por Gênero. Fonte: Resultados da pesquisa.

\section{Seus pais praticavam atividades físicas regulares quando você era jovem? * Classificação IMC * Perfil Socioeconômico-sexo} Tabulação cruzada

\% em Seus pais praticavam atividades físicas regulares quando você era jovem?

\begin{tabular}{|c|c|c|c|c|c|c|c|}
\hline \multirow{2}{*}{\multicolumn{3}{|c|}{ Perfil Socioeconômico-sexo }} & \multicolumn{4}{|c|}{ Classificação IMC } & \multirow[b]{2}{*}{ Total } \\
\hline & & & $\begin{array}{c}\text { Abaixo do } \\
\text { peso }\end{array}$ & $\begin{array}{l}\text { Levemente } \\
\text { acima do } \\
\text { peso }\end{array}$ & $\begin{array}{c}\text { Obesidade } \\
\text { grau I }\end{array}$ & Peso Ideal & \\
\hline \multirow[t]{4}{*}{ Feminino } & \multirow{3}{*}{$\begin{array}{l}\text { Seus pais praticavam } \\
\text { atividades físicas } \\
\text { regulares quando você } \\
\text { era jovem? }\end{array}$} & Não & $2,6 \%$ & $35,9 \%$ & $17,9 \%$ & $43,6 \%$ & $100,0 \%$ \\
\hline & & Não lembro. & $12,5 \%$ & $25,0 \%$ & $37,5 \%$ & $25,0 \%$ & $100,0 \%$ \\
\hline & & Sim & $18,8 \%$ & $25,0 \%$ & & $56,3 \%$ & $100,0 \%$ \\
\hline & Total & & $7,9 \%$ & $31,7 \%$ & $15,9 \%$ & $44,4 \%$ & $100,0 \%$ \\
\hline \multirow[t]{4}{*}{ Masculino } & Seus pais praticavam & Não & & $35,3 \%$ & $23,5 \%$ & $41,2 \%$ & $100,0 \%$ \\
\hline & $\begin{array}{l}\text { atividades tisicas } \\
\text { requlares quando você }\end{array}$ & Não lembro. & & $66,7 \%$ & $33,3 \%$ & & $100,0 \%$ \\
\hline & era jovem? & Sim & & & $25,0 \%$ & $75,0 \%$ & $100,0 \%$ \\
\hline & Total & & & $34,1 \%$ & $24,4 \%$ & $41,5 \%$ & $100,0 \%$ \\
\hline \multirow[t]{4}{*}{ Total } & Seus pais praticavam & Não & $1,4 \%$ & $35,6 \%$ & $20,5 \%$ & $42,5 \%$ & $100,0 \%$ \\
\hline & regulares quando você & Não lembro. & $9,1 \%$ & $36,4 \%$ & $36,4 \%$ & $18,2 \%$ & $100,0 \%$ \\
\hline & era jovem? & Sim & $15,0 \%$ & $20,0 \%$ & $5,0 \%$ & $60,0 \%$ & $100,0 \%$ \\
\hline & Total & & $4,8 \%$ & $32,7 \%$ & $19,2 \%$ & $43,3 \%$ & $100,0 \%$ \\
\hline
\end{tabular}

Quadro 6. Relação Classificação do IMC, práticas de atividades físicas dos pais, por Gênero. Fonte: Resultados da pesquisa.

A pesquisa também evidencia que $71,8 \%$ das pessoas são atendidas por estruturas para a prática de atividades físicas em seus bairros, conforme mostra o Gráfico 1.

\footnotetext{
No seu bairro, há locais públicos para a prática de atividades físicas?

103 respostas
}

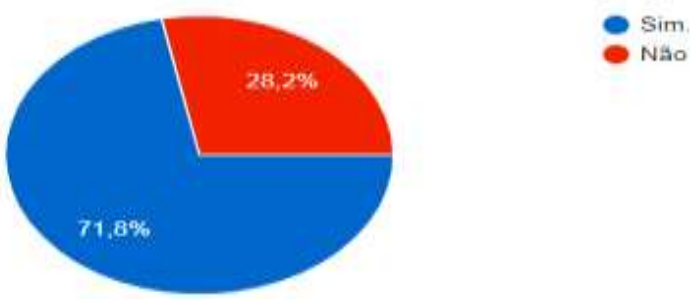

Gráfico 1. Disponibilidade de infraestrutura para o desenvolvimento de atividades físicas no bairro. Fonte: Resultados da pesquisa. 
Ou seja, as condições de oferta de infraestrutura para a prática de atividades físicas é fator relevante. Todavia, essa oferta tem sido satisfatória nos dias atuais.

A pesquisa evidencia que essas relações são relevantes. Outro aspecto a considerar, é que as estruturas públicas ofertadas para as práticas de atividades físicas são suficientes (Quadro 7).

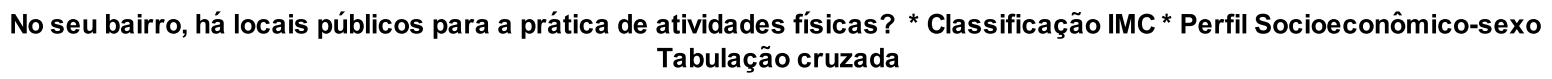

$\%$ em No seu bairro, há locais públicos para a prática de atividades físicas?

\begin{tabular}{|c|c|c|c|c|c|c|c|}
\hline \multirow{2}{*}{\multicolumn{3}{|c|}{ Perfil Socioeconômico-sexo }} & \multicolumn{4}{|c|}{ Classificação IMC } & \multirow[b]{2}{*}{ Total } \\
\hline & & & $\begin{array}{c}\text { Abaixo do } \\
\text { peso }\end{array}$ & $\begin{array}{c}\text { Levemente } \\
\text { acima do } \\
\text { peso }\end{array}$ & $\begin{array}{c}\text { Obesidade } \\
\text { grau I }\end{array}$ & Peso Ideal & \\
\hline \multirow[t]{3}{*}{ Feminino } & No seu bairro, há locais & Não & & $31,6 \%$ & $26,3 \%$ & $42,1 \%$ & $100,0 \%$ \\
\hline & de atividades físicas? & Sim. & $11,4 \%$ & $31,8 \%$ & $11,4 \%$ & $45,5 \%$ & $100,0 \%$ \\
\hline & Total & & $7,9 \%$ & $31,7 \%$ & $15,9 \%$ & $44,4 \%$ & $100,0 \%$ \\
\hline \multirow[t]{3}{*}{ Masculino } & No seu bairro, há locais & Não & & $9,1 \%$ & $36,4 \%$ & $54,5 \%$ & $100,0 \%$ \\
\hline & de atividades físicas? & Sim. & & $43,3 \%$ & $20,0 \%$ & $36,7 \%$ & $100,0 \%$ \\
\hline & Total & & & $34,1 \%$ & $24,4 \%$ & $41,5 \%$ & $100,0 \%$ \\
\hline \multirow[t]{3}{*}{ Total } & No seu bairro, há locais & Não & & $23,3 \%$ & $30,0 \%$ & $46,7 \%$ & $100,0 \%$ \\
\hline & de atividades físicas? & Sim. & $6,8 \%$ & $36,5 \%$ & $14,9 \%$ & $41,9 \%$ & $100,0 \%$ \\
\hline & Total & & $4,8 \%$ & $32,7 \%$ & $19,2 \%$ & $43,3 \%$ & $100,0 \%$ \\
\hline
\end{tabular}

Quadro 7. Relação Classificação do IMC, infraestrutura no Bairro, segmentado por Gênero. Fonte: Resultados da pesquisa.

\section{Conclusão}

Diversos fatores internos e externos envolvidos que causam a obesidade podem promover um desequilíbrio energético em longo prazo: histórico familiar de obesidade, uma vez que a doença tem influência genética e os maus hábitos alimentares, podem ser ensinados de pai para filho; fatores psicológicos, como estresse ou tédio, dificuldades de aprendizagem, e outros transtornos podem interferir no seu emocional fazendo as crianças comerem mais do que o normal. E por fim, uma criança ativa e que pratique atividade física tem menos propensão à obesidade.

Este estudo evidencia que a frequência da realização de atividades físicas desde a infância e a sua permanência na fase adulta propiciam uma vida mais saudável, a partir do indicador estabelecido. Relações envolvendo renda familiar não se mostraram relevante no processo de hábitos e cultura relacionados aos exercícios, porém, mostraram resultados desfavoráveis em relação ao IMC para baixas rendas. As respostas evidenciam que a frequência das atividades propicia também a percepção de bemestar físico e mental.

De acordo com a pesquisa foi possível compreender que o estilo de vida moderno tem interferido muito nos hábitos alimentares e na prática de exercícios físicos, assim os fatores externos como a cultura e os hábitos familiares na prática de atividade física têm papel fundamental no fenômeno da obesidade.

Os pais têm um papel muito importante a desempenhar, pois devem ensinar seus filhos a desenvolverem hábitos alimentares saudáveis e a levar um estilo de vida ativo. Como as crianças imitam o que veem, não surpreende que os comportamentos dos próprios pais estejam associados aos comportamentos das crianças e a seu peso. As crianças precisam praticar atividades físicas regulares para que o gasto calórico seja compatível com a ingestão dos alimentos.

Pais e cuidadores devem ser modelos positivos para os comportamentos alimentares e físicos das crianças. Devem moldar comportamentos alimentares saudáveis e incentivar a prática de atividade física para as crianças, de forma a reduzir seu risco de obesidade na fase adulta. A falta de conhecimento dos pais sobre nutrição saudável, comportamentos alimentares e inatividade física, pode resultar em comportamentos inadequados por parte dos filhos. Por isso é fundamental que esta reflexão também permeie o âmbito escolar, para que juntos, escola e família, busquem uma solução para o fenômeno da obesidade.

Para que a epidemia de obesidade seja enfrentada, são necessárias mudanças que promovam a atividade física. Infelizmente, a obesidade é uma doença e está cada vez crescendo mais nas famílias do Brasil. E, além da dieta, é necessário mudar os comportamentos sedentários. A prevenção da obesidade 
provavelmente não terá resultados se o ambiente da criança não for considerado. A qualidade nutricional de alimentos e bebidas servidos e vendidos nas escolas também deve ser melhorada. As crianças devem ser encorajadas a reduzir o consumo de refrigerantes e outras bebidas adoçadas com açúcar. Além de aumentar seu nível de atividade física e a reduzir sua exposição à televisão. É evidente que a prevenção deve ocorrer nas escolas, em um trabalho conjunto com os pais, de recuperar hábitos saudáveis na alimentação e no exercício físico. Este fenômeno precisa ser enfrentado como uma doença, o que muitas vezes não acontece.

De acordo com Batista Filho, Miglioli e Santos (2007), os aspectos geográficos influenciam de forma mais evidente na obesidade do que os aspectos econômicos. Ao longo do tempo observou-se o declínio da desnutrição e o aumento da obesidade como um dos maiores problemas na saúde pública, nos lugares menos industrializados a população ainda continua sofrendo com a desnutrição. OlaviUkkola (2002) afirma também que a prevalência da obesidade infantil aumentou em países industrializados durante as últimas décadas, o que não é surpreendente, pois os níveis de gastos energéticos estão diminuindo devido ao novo estilo de vida da população.

A obesidade como doença crônica é considerada atualmente uma epidemia mundial. Tanto nas pesquisas internacionais abordadas na presente pesquisa, quanto aos estudos realizados no Brasil apresentaram uma única perspectiva. Este artigo apresenta uma reflexão sobre a cultura e os hábitos familiares da prática de atividades físicas e as tendências à obesidade, com o intuito de compreender os fatores que influenciam no processo de desenvolvimento da obesidade. Os resultados apontaram que ocorre a repetição da obesidade entre as gerações e que o ambiente familiar e seus hábitos são fatores que influenciam o desenvolvimento da obesidade desde a infância. Conclui-se que há necessidade de uma ampla discussão e aplicação das soluções em torno da compreensão dos padrões relacionais estabelecidos pela família ao longo do tempo, pois estes se constituem em recursos importantes de prevenção e intervenção no contexto da obesidade. A partir desses resultados propõe-se que o tema esteja em evidência na educação como um todo.

\section{Referências}

Baratieri, R. 2005. Aspectos sociais e demográficos da obesidade. In R. S. Silva \& N. T. Kawahara (Orgs.), Cuidados pré e pós-operatórios na cirurgia da obesidade. Porto Alegre, RS: AGE.

Batista Filho, M., Miglioli, T. C., \& Santos, M. C. D. 2007. Normalidade antropométrica de adultos: o paradoxo geográfico e socioeconômico da transição nutricional no Brasil. Revista Brasileira de Saúde Materno Infantil, 7(4), 487-493.

Crossman, A., Sullivan, D. A., \& Benin, M. 2006. The family environment and American adolescents' risk of obesity as young adults. Social Science \& Medicine, 63, 2255-2267.

Fisberg, M. 1995.Obesidade na infância e adolescência. São Paulo, SP: Fundação BYK.

Fox, C. S., Pencina, M. J.; Heard-Costa, N. L., Shrader, P., Jaquish, C., O’Donnell, C. J., ... Agostino, R. B. 2013. Trends in the Association of Parental History of Obesity over 60 Years. Obesity, 22(3), 919-924

Geremia, R., Cimadon, H. M. S., Souza, W. B., \& Pellanda, L. C. 2015. Childhood overweight and obesity in a region of Italian immigration in Southern Brazil: a cross-sectional study. Italian Journal of Pediatrics, $41(1), 28$

Glasenapp, D. 2008. Obesidade Infantil: a educação como referência. Joinville, SC: Revista Univille.

Goldenberg, M. \& Ramos, M. S. 2007. A civilização das formas: o corpo como valor. In M. Goldenberg (Org.), Nu e vestido: dez antropólogos revelam a cultura do corpo carioca. Rio de Janeiro, RJ: Record.

Instituto Brasileiro de Geografia e Estatística[IBGE]. 2015. Pesquisa Nacional de Saúde 2013: Ciclos de Vida. Rio de Janeiro. Disponível em: http://biblioteca.ibge.gov.br/visualizacao/livros/liv94522.pdf. Acesso em: 02 fev. 2020.

Kelly, G. E., Murrin, C., Viljoen, K., O'Brien, J., \& Kelleher, C. 2014. Body mass index is associated with the maternal lines but height is heritable across family lines in the Lifeways Cross-Generation Cohort Study. BMJ Open, 4(12), e005732.

Mascarenhas, L. P. G., Modesto, M. J., Amer, N. M., Boguszewski, M. C. S., de Lacerda Filho, L., \& Prati, F. S. 2013. Influência do excesso de peso dos pais em relação ao sobrepeso e obesidade dos filhos. Pensar a Prática, 16(2). 
Ministérioda Saúde. (2014). Perspectivas e desafios no cuidado às pessoas com obesidade no SUS: resultados do laboratório de inovação no manejo da obesidade nas redes de atenção à saúde. Brasília, DF: Ministério da Saúde.

Minuchin, S. 1990.Famílias: funcionamento e tratamento (2a ed.). Porto Alegre, RS: Artes Médi.

Moira, A. P., Power, C., \&Li, L. 2010. Changing Influences on Childhood Obesity: A Study of 2 Generations of the 1958 British Birth Cohort. American Journal of Epidemiology, 171(12), 1289-1298.

OlaviUkkola, C. B. 2002.Fatores Genéticos e obesidade infantil. São Paulo, SP: Anais Nestlé.

Organização Mundial da Saúde [OMS]. 2015. Nota descriptiva $n^{\circ}$ 311: Obesidad y Sobrepeso. Disponível em: http://www.who.int/mediacentre/factsheets/fs311/es/. Acesso em: 05 out. 2016.

Vasques, F., Martins, F. C., \& Azevedo, A. P. 2004. Aspectos psiquiátricos do tratamento da obesidade. Revista Psiquiatria Clínica, 31(4), 195-198.

\section{Minicurrículo}

Liana Fátima Fuga. Possui graduação em Educação Física pela Escola Superior de Educação Física (1982) e mestrado em Educação pela Universidade Metodista de Piracicaba (1992). Tem experiência na área de Educação e em Educação Física. Atua no Centro de Ciências Sociais Aplicadas na Unioeste-Universidade Estadual do Oeste do Paraná- no Campus de Cascavel e no Hospital Universitário.

Como citar: Fuga, L.F. 2020.A cultura e os hábitos familiares da prática de atividades físicas e as tendências à obesidade. Pubsaúde, 3, a023. DOI: https:// dx.doi.org/10.31533/pubsaude3.a023

Recebido: 26 fev. 2020.

Revisado e aceito: 10 mar. 2020.

Conflito de interesse: os autores declaram, em relação aos produtos e companhias descritos nesse artigo, não ter interesses associativos, comerciais, de propriedade ou financeiros que representem conflito de interesse.

Licenciamento: Este artigo é publicado na modalidade Acesso Aberto sob a licença Creative Commons Atribuição 4.0 (CC-BY 4.0). 